\title{
Hydrocephalic Dementia: Revisited with Multimodality Imaging and toward a Unified Imaging Approach
}

\author{
Sandhya Mangalore ${ }^{1, \odot}$ Sriharish Vankayalapati ${ }^{1, \odot}$ \\ ${ }^{1}$ Division of Neuroradiology, Department of Neuroimaging and \\ interventional Radiology, National Institute of Mental Health and \\ Neurosciences, Bengaluru, India
}

Arun Kumar Gupta ${ }^{1, \odot}$

\begin{abstract}
Address for correspondence Sandhya Mangalore, MD, DM, Division of Neuro-radiology, Department of Neuroimaging and interventional Radiology, National Institute of Mental Health and Neurosciences, Bengaluru 560029, India (e-mail: drsandym@gmail.com).
\end{abstract}

\begin{abstract}
Keywords

- molecular imaging

- positron emission tomography-

- magnetic resonance imaging

- dementia

- multimodality approach

- normal pressure hydrocephalus

Objective Overlap of normal pressure hydrocephalus (NPH) and pathology proven cases of dementia is known. The objective of this paper is to correlate both the clinical and multimodality imaging findings in patients with imaging diagnosis NPH and give a hypothesis for association of clinical findings.

Methods This is a retrospective observational analysis of 13 cases patients who were referred to molecular imaging center for imaging in 2016 to 2019, and they were divided into four groups based on structural imaging findings. Group 1 had magnetic resonance imaging (MRI) findings of diffuse effacement of sulcal spaces (DESH) and flow void, whereas Group 4 had none of these two. Group 3 had MRI findings of DESH but no flow void, and Group 2 had flow void but no DESH. Clinical presentation, MRI-PET findings of four groups are assessed.

Results Groups with presence of flow void showed hypometabolism in the medial frontal and medial temporal lobe. Groups with presence of DESH has effacement of parietal sulci showed parietal hypo metabolism with clinical presentation $A D /$ mixed dementia and absence of parietal effacement showed FTD-like presentation. Groups without flow void or DESH showed only mild medial temporal hypometabolism and presented with classical signs of NPH. ASL perfusion changes are in correlation with metabolism on positron emission tomography (PET)-MRI.

Conclusion This study has led us to hypothesize the lack of outflow of brain protein and their deposition in parenchyma based on pressure gradient would be easier explanation to go with cluster of findings. MR-PET and other investigations each had different specificity and sensitivity and different pattern of presentation.
\end{abstract}

\section{Introduction}

Normal pressure hydrocephalus (NPH) is a syndrome characterized by the triad of gait disturbance, mental deterioration, and urinary incontinence, associated with ventriculomegaly and normal cerebrospinal fluid (CSF) pressure. ${ }^{1}$ The clinical presentation (triad) may be atypical or incomplete and/or mimicked by other diseases. ${ }^{2}$ Hence, there is need for supplementary tests such as CSF tap-tests and computed tomography (CT) or magnetic resonance imaging (MRI) to confirm
DOI https://doi.org/ $10.1055 / \mathrm{s}-0041-1726614$ ISSN 0976-3147
(C)2021. Association for Helping Neurosurgical Sick People.

This is an open access article published by Thieme under the terms of the Creative Commons Attribution-NonDerivative-NonCommercial-License, permitting copying and reproduction so long as the original work is given appropriate credit. Contents may not be used for commercial purposes, or adapted, remixed, transformed or built upon. (https://creativecommons.org/licenses/by-nc-nd/4.0/).

Thieme Medical and Scientific Publishers Pvt. Ltd. A-12, 2nd Floor, Sector 2, Noida-201301 UP, India 
diagnosis and guiding patient management, particularly to predict post shunt outcome.

Overlap of NPH and pathology proven DLB-PSP-PD is known. ${ }^{3}$ Many papers set aside the imaging features like periventricular atrophy mimicking the imaging diagnosis of NPH, but histopathology which is the gold standard showed neurodegeneration with final diagnosis written off as DLB or PD and suggest that there will be no improvement after shunt.

In our experience, we come across patients showing incidental findings of NPH on imaging and FTD- or AD-like picture on PET with extremely varied clinical presentations.

The objective of this paper is to correlate both the clinical and multimodality imaging findings in patients with imaging diagnosis NPH and give a hypothesis for association of findings.

Our hypothesis in this study is that chronic hydrocephalus has caused pressure on the brain and faulty drainage, which in turn alters the perfusion and metabolism in the brain. This may explain the varied clinical presentation in NPH.

\section{Materials and Methods}

Thirteen cases who were referred to molecular imaging center for imaging between 2016 and 2019 with various clinical conditions and incidental imaging findings of NPH are included in the study.
For each selected case, the findings on multimodality imaging and their clinical presentation are noted and divided into four groups based on presence or absence of MRI findings, and correlated each subgroup with metabolic or perfusion changes. The finding of NPH in all four groups were based on imaging criteria as proposed earlier ${ }^{1}$ and include sylvian fissure prominence (DESH), parietal sulcal effacement, disproportionate ventricular enlargement, and presence flow void at fourth ventricle level.

Group 1 had MRI findings of DESH and flow void, whereas Group 4 had none of these two. Group 3 had MRI findings of DESH but no flow void, and Group 2 had flow void but no DESH.

On FDG-PET in various groups, the metabolic pattern of areas showing hypometabolism as given below was used to phenotype: medial frontal/anterior cingulate/lateral frontal for (behavioral variant of frontotemporal dementia), precuneus mid sag/high parietal/posterior cingulated/medial temporal lobe for $(A D)$, temporal pole/lateral temporal lobe for (SVFTD), and occipital for (diffuse lewy body dementia), mix of all is mixed pattern.

\section{Results}

MRI imaging markers was correlated with clinical symptoms, and metabolic changes in different groups are summarized in (-Table 1) or increased pressure to cause pressure changes on underlying cortical parenchyma.

Table 1 Clinical details and investigations in four groups

\begin{tabular}{|c|c|c|}
\hline & Clinical details & MRI pet imaging and other investigations \\
\hline \multicolumn{3}{|c|}{ Group 1: flow void positive and DESH positive (- Fig. $\mathbf{1}$ ) } \\
\hline \multicolumn{3}{|c|}{ Group 1 with absence of parietal effacement } \\
\hline Case 4 & FTD & NPH-FTD \\
\hline \multicolumn{3}{|c|}{ Group 1 with presence of parietal effacement } \\
\hline Case 3 & ? AD & $\begin{array}{l}\text { NPH: mixed pattern/? AD } \\
\text { Autoimmune paraneoplastic negative }\end{array}$ \\
\hline Case 10 & AD like & NPH and mixed dementia pattern \\
\hline Case 11 & $\mathrm{NPH}$ & $\begin{array}{l}\text { NPH-mixed pattern } \\
\text { Post shunt: parietal sulci not effaced and metabolism recovered } \\
\text { Clinically improved in parietal lobe function } \\
\text { Parietal lobe may be the first area to be affected by high CSF } \\
\text { pressure and CSF volumes. }\end{array}$ \\
\hline \multicolumn{3}{|c|}{ Group 2: flow void positive and DESH negative (- Fig. 2) } \\
\hline \multicolumn{3}{|c|}{ Group 2 with Absence of parietal effacement } \\
\hline Case 5 & $\mathrm{NPH}$ & NPH-FTD \\
\hline Case 13 & Parkinson's disease dementia & NPH-FTD \\
\hline \multicolumn{3}{|c|}{ Group 2 with absence of parietal effacement } \\
\hline Case 1 & Anxiety and delirium & $\begin{array}{l}\text { NPH-AD, granuloma } \\
\text { subdural hematoma }\end{array}$ \\
\hline Case 8 & NPH-rapid onset- paraneoplastic & $\begin{array}{l}\text { NPH: mixed pattern } \\
\text { ANA profile-CENP-B, DsDNA positive }\end{array}$ \\
\hline Case 9 & $\begin{array}{l}\mathrm{K} / \mathrm{C} / \mathrm{O} \text { of squamous cell carcinoma with } \\
\text { imbalance and features of raised ICP }\end{array}$ & NPH: mixed pattern \\
\hline
\end{tabular}


Table 1 (Continued)

\begin{tabular}{|c|c|c|}
\hline & Clinical details & MRI pet imaging and other investigations \\
\hline Case 12 & FTD + PD/DLBD & NPH: FTD/AD like \\
\hline \multicolumn{3}{|c|}{ Group 3: flow void negative and DESH positive (-Fig. 3 ) } \\
\hline Case 6 & $\mathrm{NPH}$ & $\begin{array}{l}\text { NPH: Mixed pattern } \\
\text { Paraneoplastic antibody positive: Zlc4, TPO, anti-Tg }\end{array}$ \\
\hline Case 7 & BVFTD/?DLBD & $\mathrm{NPH}-\mathrm{AD}$ \\
\hline \multicolumn{3}{|c|}{ Group 4: flow void negative and DESH negative ( - Fig. 4 ) } \\
\hline Case 2 & DLBD & NPH: mixed pattern \\
\hline \multicolumn{3}{|c|}{ 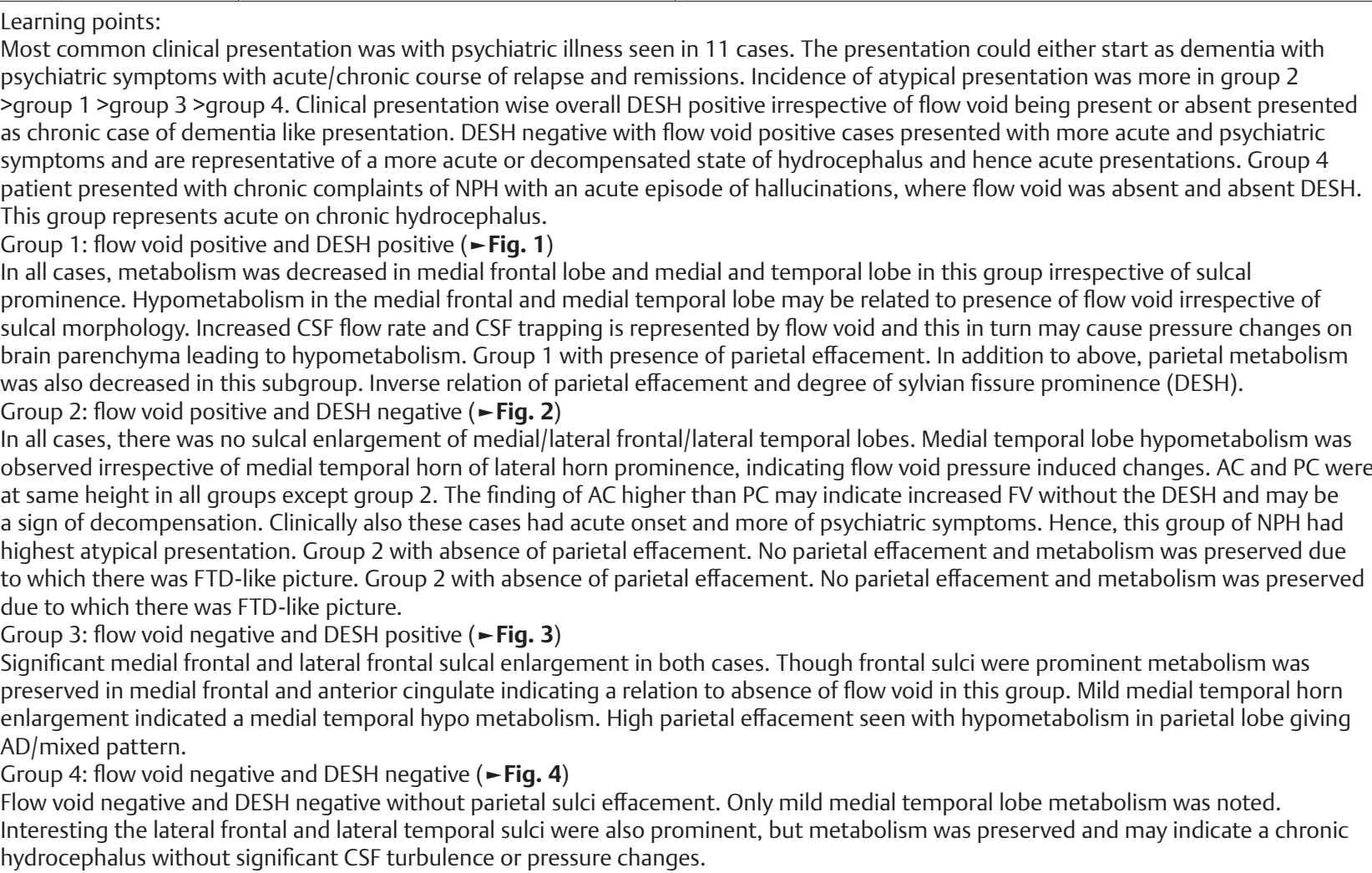 } \\
\hline
\end{tabular}

Abbreviations: AD, Alzheimer's dementia; BVFTD, behavioral variant of frontotemporal dementia; DESH, diffuse effacement of sulcal spaces; DLBD, diffuse lewy body dementia; FTD, frontotemporal dementia; NPH, normal pressure hydrocephalus.

\section{Discussion}

Comorbid NPH and another neurodegeneration are known entity. ${ }^{2} \mathrm{NPH}$ is an imaging entity with lack of specific clinical and histopathology biomarker. Hence is often ignored without an intervention and with varied treatment outcomes. A study on association between mechanical effects and behavior by Gustafson and Hagberg ${ }^{4}$ suggest prolonged mechanical stress cause a more irreversible damage on brain, specifically frontal temporo-limbic and diencephalic-hypothalamic axis. Our study results were very similar and these areas overlap with areas involved AD, FTD, PD, etc. In a paper by Roberts et al, ${ }^{5}$ he proposed amyloid deposition could be due to faulty CSF drainage.

Group 2 in our study reflects this group with PET showing more hypometabolism in group 2 than group 1 and having more acute clinical presentation. High parietal tightness indicates shunt responsiveness ${ }^{6}$ and indicates increased pressure in ventricles. Postdiagnostic LP likely there is a recoil of the brain tissue with metabolism being restored. ASL which is a perfusion marker has shown decreased global perfusion and post shunt improvement in $\mathrm{NPH} .{ }^{7}$ ASL or FDG-PET may be important biomarkers for planning a treatment.

MRI markers for mechanoelastic changes are parietal effacement, which correlated directly with hypometabolism and clinical symptoms and presence of flow void. When only DESH was present, supratentorial sulcal prominence was noted suggestive of CSF trapped without secondary pressure changes. All the cases of NPH had temporal hypometabolism.

In Group 1 and 2 hypometabolism/hypoperfusion and structural MRI findings are not linearly correlated. Group 3 and 4, which had absent flow void, had temporal hypometabolism and correlated with structural MRI. Future studies with elastography in group 1 and 2 would be the way to go for understanding more on hydrocephalic dementia, its role in proteinopathy and role of early diagnosis markers such as CSF a $\beta$ protein level for prognostication. 


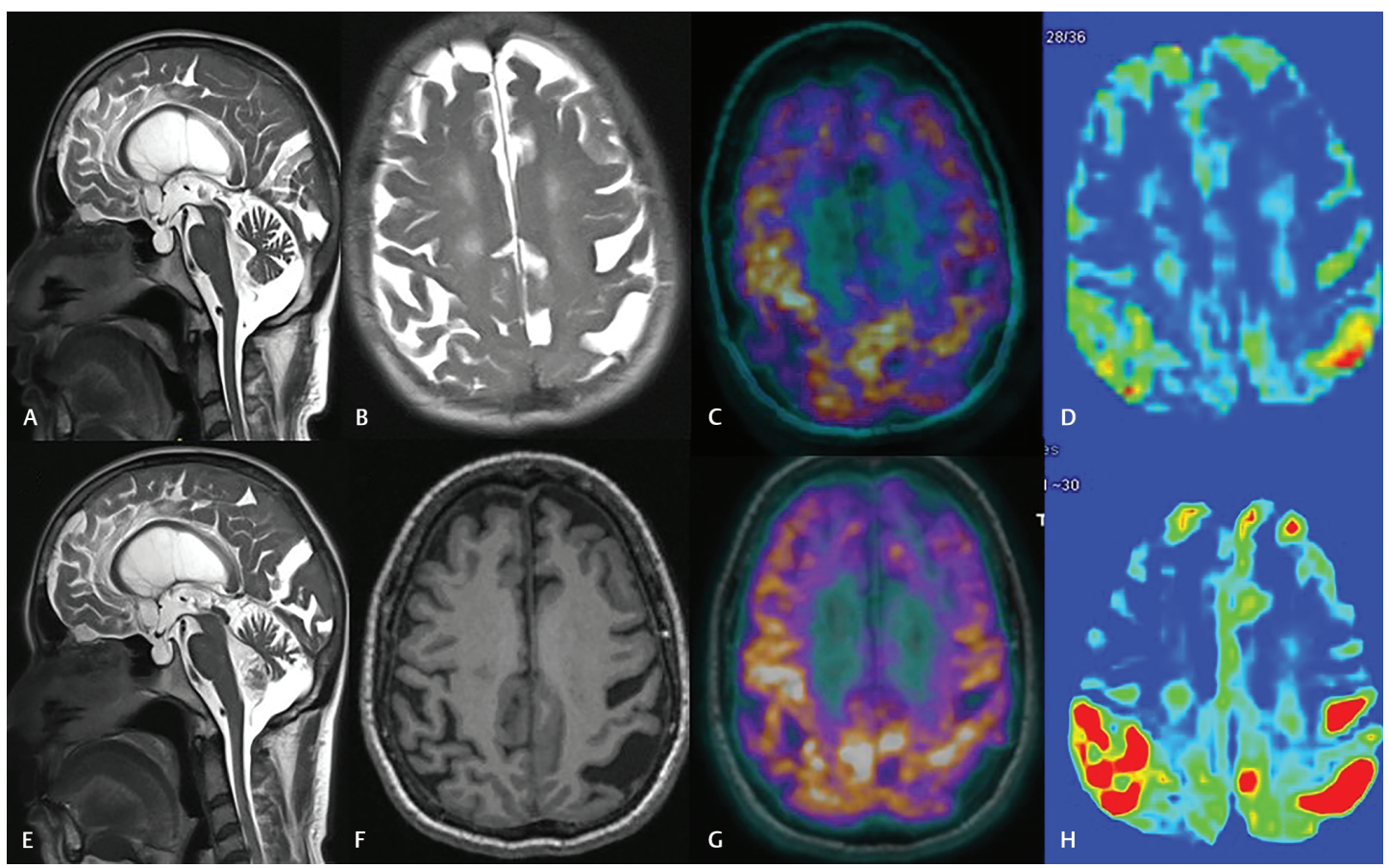

Fig. 1 This is case 11 is a representative case for group 1: magnetic resonance imaging and metabolic and perfusion changes pre-tap (A-D) and below postdiagnostic lumbar tap (E-H). Represented by Sagittal (A, E) T2w image, axial (F) T2w image, axial (F) T1w images, axial $(\mathbf{C}, \mathbf{G})$ PET images and ASL (D, H) images. Structural MRI shows mild reduction in sulcal dilatation in parietal region post tap. ASL and PET shows improved parietal perfusion and metabolism postdiagnostic LP with clinical improvement in parietal lobe function. Frontal lobe perfusion and metabolism did not change. ASL, arterial spin labeling; PET, positron emission tomography.

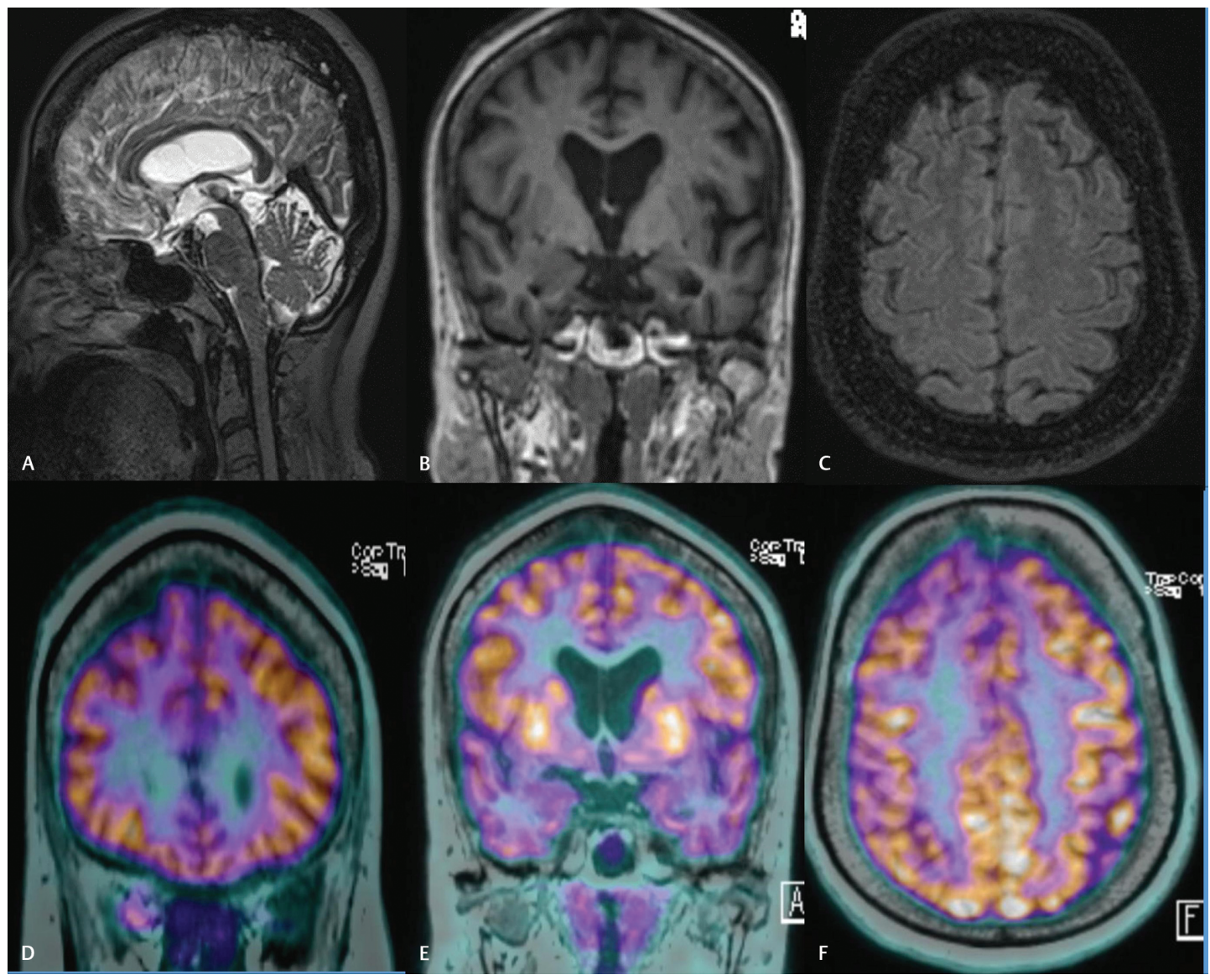

Fig. 2 This is case 5 is a representative case for group 2: represented by sagittal (A) T2w image, coronal (B) T1w image, axial (C) flair image, coronal (D, E) and axial (F), PET images. Magnetic resonance imaging shows lack of parietal effacement with flow void positive and DESH negative. PET is showing frontal and temporal hypo metabolism suggestive of frontotemporal dementia. ASL, arterial spin labeling; DESH, diffuse effacement of sulcal spaces; PET, positron emission tomography. 


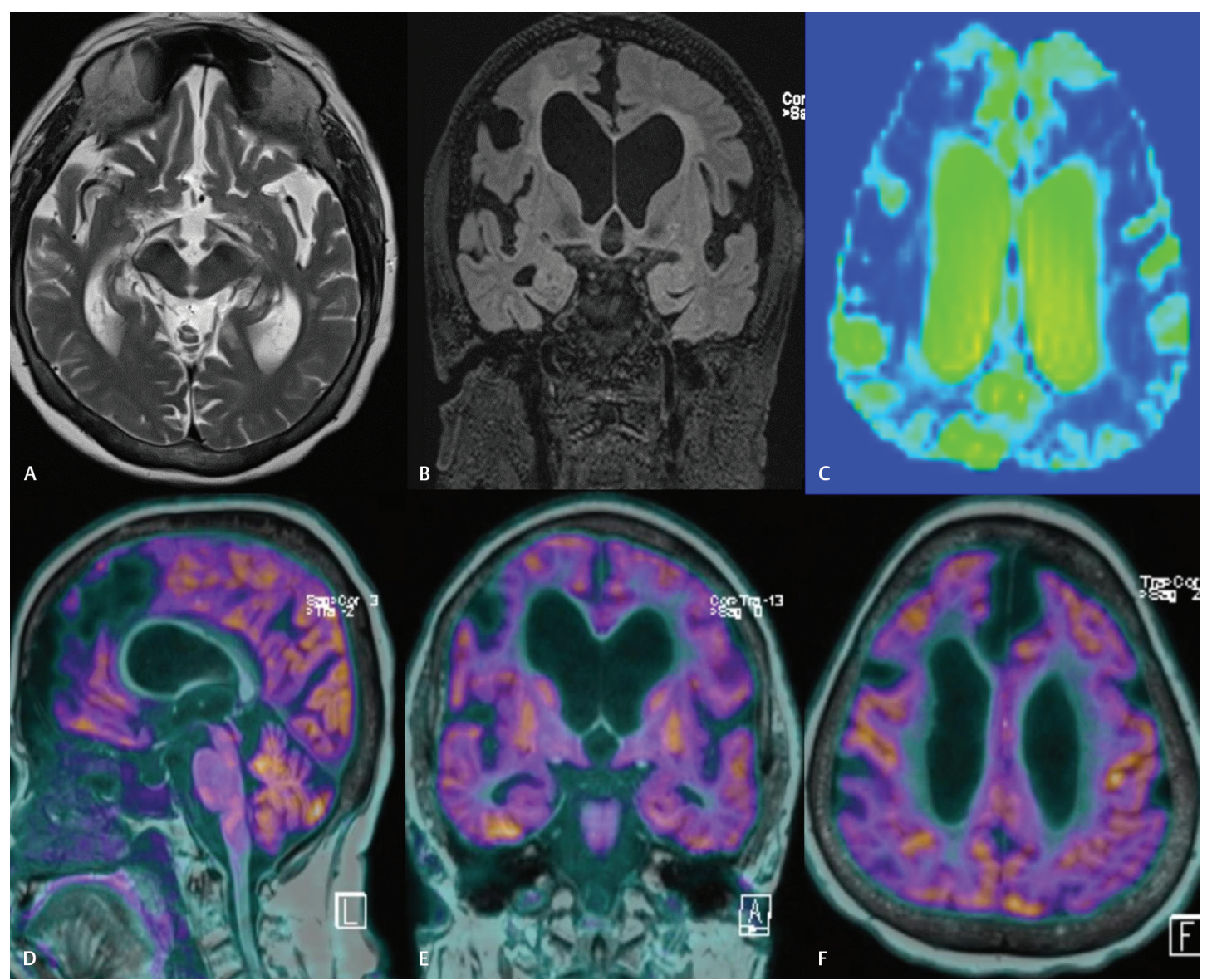

Fig. 3 This is case 6 is a representative case for group 3: represented by axial (A) T2w image, coronal (B) flair image, axial (C) ASL image, sagittal (D) coronal (E) and axial (F), PET images. (A-C) Flow void negative and DESH. There is dilatation of frontal and temporal sulci with effaced parietal sulci on MRI with flow void negative and DESH and hypoperfusion in parietal lobes. PET is showing hypometabolism in parietal and temporal lobes suggestive of mixed dementia. ASL, arterial spin labeling; DESH, diffuse effacement of sulcal spaces; PET, positron emission tomography.

A model of mechanical effects and biological changes it causes in terms of perfusion, protein deposition pattern needs to be developed. Amplitude phase MRI ${ }^{8}$ (aMRI) is one such technique. Building this link may shed light between MRI imaging NPH findings and histopathology pattern observation. ${ }^{9}$ Studies $^{8}$ using amplitude (aMRI) to measure shear stress and strain ${ }^{10}$ in NPH are lacking.

Simultaneous magnetic resonance electrography (MRE) and functional MRI have shown that brain stiffening and softening as a result of neuromechanical coupling occurrence, wherein the brain softens at areas of increased neurohemodynamic coupling during a task. This application proves that perfusion neuronal activity and mechanical properties of brain are coupled

FDG-PET scans in pure NPH have shown decreased global metabolism and/or a heterogeneous mixed pattern of hypometabolism ${ }^{11}$. Amyloid PET scans in NPH patients showed increased brain amyloid deposition ${ }^{12}$ in cases with NPH associated with decreased CSF levels of amyloid beta (a BETA) and an increased tau thereby reflecting decreased clearance of brain amyloid via CSF. Studies show amyloid has a protective role and induces synaptic plasticity. ${ }^{5}$ So, by measuring CSF levels of a BETA may give an idea of amyloid deposition and help plan a shunt to reverse dementia. ${ }^{13}$

The nonlinear changes between metabolic changes and structural MRI and the symptoms depended on amount of pressure that cortical area was facing need to be correlated with MRE studies, and ASL studies as has been highlighted and model developed in our previous study ${ }^{14}$.

Our study is unique as it attempts to correlate with simultaneous imaging the structural metabolic changes in NPH.

So, we hypothesize that chronic hydrocephalus with periods of relapse and remission has caused pressure on the brain, and the faulty CSF drainage has caused the deposition of toxins such as amyloid, synucleins, and tau proteins unlike other dementia which are genetically programmed. Altered CSF pressure and drainage of toxic proteins in turn lead to varied metabolic and perfusion changes.

In conclusion, overall on correlation of clinical presentation, MR PET and other investigations each had different specificity and sensitivity and different pattern of presentation. All the above cases were atypical cases and clinical and imaging correlation was different to come to a definite conclusion. Hence, this study starts with NPH as starting 


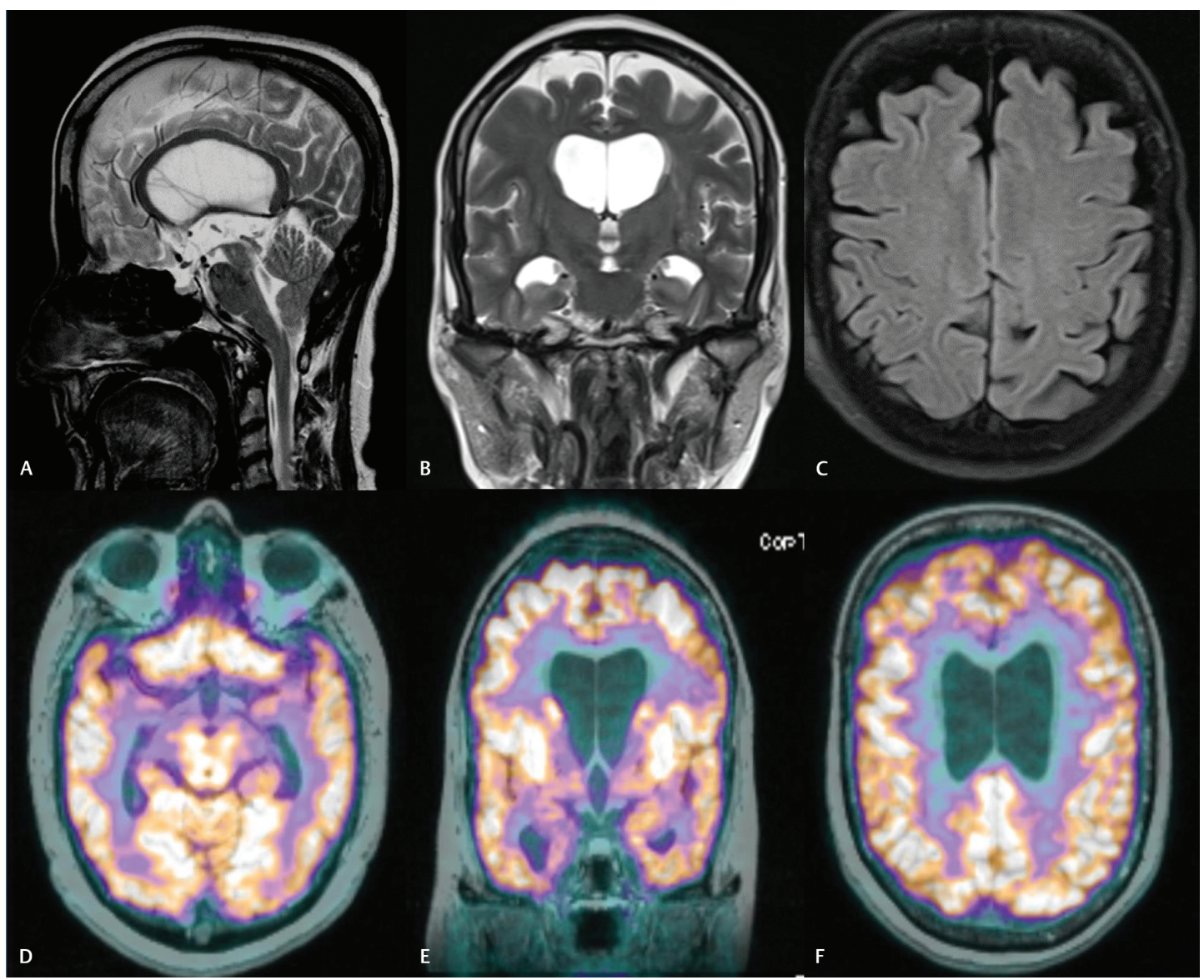

Fig. 4 This is a case 2 is a representative case for group 4: represented by sagittal (A) and coronal (B) T2w image, axial (C) flair image, axial (D, F) and coronal (E) PET images. Magnetic resonance imaging showed dilatation of frontal parietal sulci and medial temporal horn with flow void negative and DESH negative. PET shows hypometabolism in medial temporal lobe only suggestive of normal pressure hydrocephalus. ASL, arterial spin labeling; PET, positron emission tomography.

point and concluding that clinical presentation, serology, and metabolic changes may be different spectra of NPH/chronic hydrocephalus. Each modality has its own sensitivity and specificity, and no modality is absolute, and hence, a thorough workup should be advocated for atypical presentations.

\section{Funding \\ None.}

\section{Conflict of Interest}

None declared.

\section{References}

1 Damasceno BP. Neuroimaging in normal pressure hydrocephalus. Dement Neuropsychol 2015;9(4):350-355

2 Alisky J. Normal pressure hydrocephalus co-existing with a second dementia disorder. Neuropsychiatr Dis Treat 2008; 4(1):301-304

3 Starr BW, Hagen MC, Espay AJ. Hydrocephalic parkinsonism: lessons from normal pressure hydrocephalus mimics. J Clin Mov Disord 2014;1(1):2
4 Gustafson L, Hagberg B. Recovery in hydrocephalic dementia after shunt operation. J Neurol Neurosurg Psychiatry 1978; 41(10):940-947

5 Roberts GW, Gentleman SM, Lynch A, Murray L, Landon M, Graham DI. Beta amyloid protein deposition in the brain after severe head injury: implications for the pathogenesis of Alzheimer's disease. J Neurol Neurosurg Psychiatry 1994; 57(4):419-425

6 Narita W, Nishio Y, Baba T, et al. High-convexity tightness predicts the shunt response in idiopathic normal pressure hydrocephalus. AJNR Am J Neuroradiol 2016;37(10):1831-1837

7 Virhammar J, Laurell K, Ahlgren A, Cesarini KG, Larsson EM. Idiopathic normal pressure hydrocephalus: cerebral perfusion measured with pCASL before and repeatedly after CSF removal. J Cereb Blood Flow Metab (Nihongoban)

8 Holdsworth SJ, Rahimi MS, Ni WW, Zaharchuk G, Moseley ME. Amplified magnetic resonance imaging (aMRI) Magn Reson Med 2016;75(6):2245-2254

9 Budday S, Ovaert TC, Holzapfel GA, Steinmann P, Kuhl E. Fifty shades of brain: a review on the mechanical testing and modeling of brain tissue. Arch Computat Methods Eng2019; 27:1187-1230 
418 Multimodality Imaging and Unified Imaging Approach Mangalore et al.

10 Murphy MC, Jones DT, Jack CR Jr, et al. Regional brain stiffness changes across the Alzheimer's disease spectrum. Neuroimage Clin 2015;10:283-290

11 Townley RA, Botha H, Graff-Radford J, et al. 18F-FDG PET-CT pattern in idiopathic normal pressure hydrocephalus. Neuroimage Clin 2018;18:897-902

12 Jang H, Park SB, Kim Y, et al. Prognostic value of amyloid PET scan in normal pressure hydrocephalus. J Neurol 2018; 265(1):63-73
13 Mc M, Dt J, Cr J, et al. Regional brain stiffness changes across the alzheimer's disease spectrum. Neuroimage Clin 10.1016/j. nicl.2015.12.007

14 Mangalore S, Rakshith S, Srinivasa R. Solving the riddle of "Idiopathic" in idiopathic intracranial hypertension and normal pressure hydrocephalus: an imaging study of the possible mechanisms - Monro-Kellie 3.0. Asian J Neurosurg 2019; 14(2):440-452 